\title{
Hypothalamic and limbic system activation during salt arousal of drinking'
}

An attempt was made with reasonable success to record multiunit neural activity chronically from rat hypothalamus and various limbic structures during the salt arousal of drinking. Increases in activity which were usually recorded occurred in the hypothalamus, medial habenular nucleus, lateral septal nucleus, diagonal bands of broca, cingulate cortex, caudate nucleus, fomix, and thalamus.

In the laboratory it is relatively easy to make a rat drink water by increasing its extracellular ionic concentration (salt arousal of drinking). Andersson (1952) was one of the first to emphasize the role of concentration or osmotic sensitive cells in the hypothalamus in the mediation of drinking. Considerable evidence has accumulated (Wayner, 1964) which implicates many parts of the brain in drinking and the regulation of body water. Recent work of Oomura, Ooyama, Yamamoto, \& Naka (1967), Iki (1964) Ishikawa, Koizumi, \& Brooks (1966), and Joynt (1964) demonstrates direct modulation of unit activity in the hypothalamus and various limbic structures of the cat related to drinking. Little, if any, electrophysiological data is available on the rat under similar conditions. The purpose of the present experiment was to record multiunit activity in the hypothalamus and various limbic structures of the rat brain during salt arousal of drinking. Because of the complications introduced by the use of an anesthetic (Oomura, Ooyama, Yamamoto, \& Naka, 1967), an attempt was made to obtain these data in the normal wakeful animal through chronically implanted electrodes and cannulas. Method

A total of 68 male hooded rats 14 months in age selected according to predetermined stereotaxic criteria were used in this study. Under ether anesthesia, the left common carotid artery was cannulated with No. 10 polyethylene tubing (filled with normal saline plus heparin) which was connected to a syringe which was continuously driven at a slow rate. Flexible electrodes, one mil stainless steel wire coated four times with Formex, were driven slowly by means of a micromanipulator through a hole in the skull to predetermined positions in the brain; and if the unit activity were acceptable, then Acralite dental cement was poured around the electrode and stainless steel screws (previously fastened to the skull). One screw served as an indifferent electrode and to support a connector to Microdot (50-3804) wire, required to reduce lead noise. Three to four electrodes were inserted into the left half of the brain of each animal.
Electrical recording was accomplished by means of Tektronix 122 preamplifiers and four trace oscilloscope, and permanent records were obtained on $35 \mathrm{~mm}$ film by means of a Grass kymograph camera. Records were inspected visually by projecting the film onto a large screen. Number of multiunit or single unit spikes were obtained by counting the spikes in representative samples of the records at 1-min. intervals during the course of an experiment. All discernible spikes in a sample were counted. The animal was placed in a small restricted enclosure and maintained continuously, both the cannula and electrical leads were unwound manually when necessary. Results were obtained about $24 \mathrm{hr} .-36 \mathrm{hr}$. after the electrodes were implanted. An experimental trial consisted of a single injection of $0.05 \mathrm{cc}$ of distilled water of $15 \% \mathrm{NaCl}$ through the indwelling carotid cannula at the rate of $0.05 \mathrm{cc} / \mathrm{min}$. The position of the electrode tip was verified by marking the location with the prussian blue method and counterstaining tissue sections with saffranin.

Results

Although in every case each electrode displayed unit activity at the time it was permanently cemented into position, most of them were relatively inactive by the end of $24 \mathrm{hr}$. when the animals' motor activity appeared to have returned to normal patterns. Another limiting factor was maintenance of a functional cannula. Also, the error involved in attempting to place the electrodes in a predetermined structure when driving a flexible electrode was large. However, occasional success warranted continuing until a suffi-

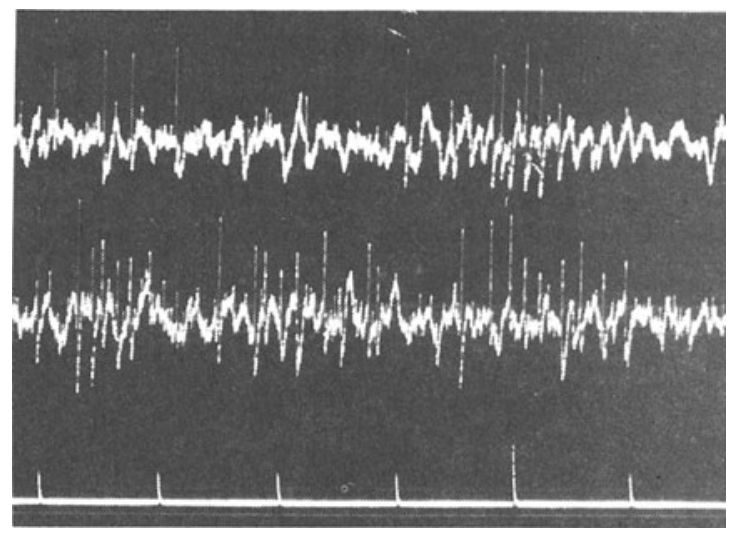

Fig. 1. Multiunit activity recorded from medial habenular nucleus before (top tracing) and 6 min. after (lower tracing) $0.05 \mathrm{cc} 15 \%$ NaCl was injected into the carotid artery. Time marks, each division 0.1 sec. 


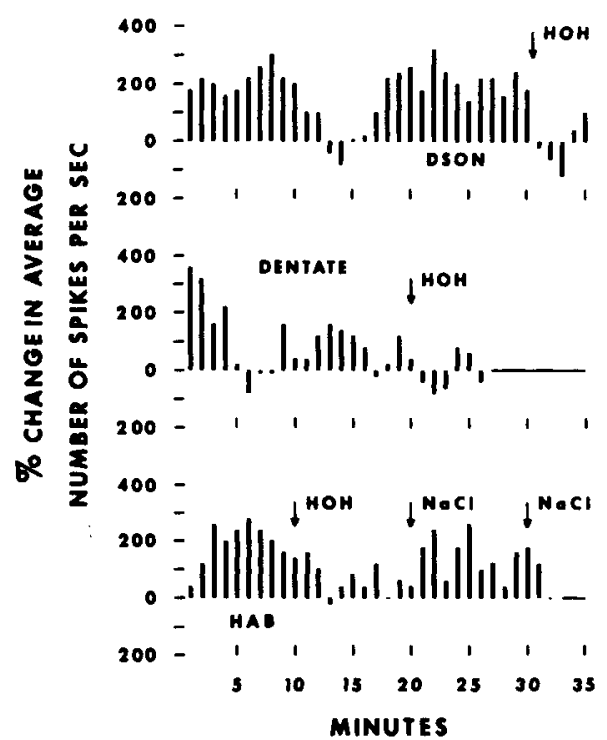

Fig. 2. Per cent change in multiunit activity (spikes per sec.) recorded dorsal to the supraoptic nucleus (DSON), in the dentate gyrus (DENTATE), and in the medial habenular nucleus (HAB) following injection of $0.05 \mathrm{cc} 15 \% \mathrm{NaCl}$ and water (HOH) into the carotid artery.

cient number of animals had been examined.

Figure 1 is a sample of typical multiunit activity recorded in the medial habenular nucleus in the normal wakeful state (top tracing) and $6 \mathrm{~min}$. (see Fig. 2) after the beginning of the administration of 0.05 cc $15 \% \mathrm{NaCl}$ (bottom tracing). Figure 2 illustrates the changes observed in three experiments on different animals. The ordinate represents percent change in the number of discernible spikes per sec. Samples of records (10-sec. duration) were obtained at 1-min. intervals following the beginning of the administration of $0.5 \mathrm{cc}$ of $15 \% \mathrm{NaCl}$. The hypertonic saline was administered during the first min. Subsequent injections are indicated by arrows. Horizontal line indicates no change as compared to the baseline. Data in the top part of Fig. 2 was obtained in a region just dorsal to the supraoptic nucleus. Although the $\mathrm{NaCl}$ increased the activity, it diminished spontaneously within $13 \mathrm{~min}$., then increased again, and was decreased by the injection of $0.05 \mathrm{cc}$ of distilled water.
Data from the dentate gyrus (middle portion of Fig. 2) shows a relatively brief increase in activity, a decrease, then a gradual increase and decrease again. In the hypothalamus and medial habenular nucleus (lower portion of Fig. 2) both the frequency of individual cells and the number of cells which respond usually increase. Water reversed the effect and activity was reduced to its original level. In the medial habenular nucleus the third injection of hypertonic saline appeared to inhibit activity. A reduction in neural activity was usually observed following repeated injections. Most reliable increases were found following single injections. Reduction in activity is related to the inhibition of certain units and has been observed before (Joynt, 1964) under these conditions. Latency is difficult to measure; obvious changes were observed at times to occur within $10 \mathrm{sec}$. after the injection was started.

Other parts of the brain in which an increase in activity occurred during salt arousal were lateral septal nucleus, diagonal band of broca, cingulate cortex, anterior portion of the caudate nucleus, and portions of the fornix and thalamus. The question as to which portions or structures of the brain did not respond cannot be answered at this time because, with this technique, it proved impossible to adequately sample large regions of the brain. Also, multiunit activity is difficult to quantify.

\section{References}

Anderson, B. Polydipsia caused by intrahypothalamic injections of hypertonic NaCl-solutions. Experientia. 1952, 8, 157-158.

Iki, M. Firing responses of neurons in the hypothalamic feeding center to variable electrical and chemical stimulations. Acta. Med. Univ. Kagoshima. 1964, 6, 155-175.

Ishikawa, T., Koizumi, K., \& Brooks, C. Activity of supraoptic nucleus neurons of the hypothalamus. Neurology, Minneapolis. $1966,16,101-106$.

Joynt, R. J. Functional significance of osmosensitive units in the anterior hypothalamus. Neurology. Minneapolis. 1964, 14, 584-590.

Oomura, Y., Ooyama, H., Yamamoto, T., \& Naka, F. Reciprocal relationship of the lateral and ventromedial hypothalamus in the regulation of food intake. Physiol. Behav., 1967, 2, (in press). Wayner, M. J. (Ed.) Thirst in the regulation of body water. London, Pergarnon: 1964,570 .

\section{Note}

1. Supported by a grant from the National Science Foundation No. GB-2119. 